\title{
Percepción de Desigualdad Económica en Chile: Medición, Diferencias y Determinantes
}

\author{
Perception of Economic Inequality in Chile: \\ Measurement, Differences and Determinants
}

\author{
Juan Carlos Castillo, Daniel Miranda y Diego Carrasco \\ Pontificia Universidad Católica de Chile
}

\begin{abstract}
Si bien hasta ahora se han utilizado diferentes indicadores de desigualdad económica percibida en investigaciones basadas en encuestas, no existen estudios específicos que comparen estos indicadores. Usando datos de la encuesta representativa nacional ISSP $2009(N=1.505)$, la investigación comparó 3 medidas de percepción de desigualdad en Chile y analizó la influencia de predictores de estatus, posición política y evaluación de justicia salarial en las variables perceptuales, mediante métodos de correlación y regresión. De acuerdo a los resultados, los indicadores perceptuales se encuentran débilmente asociados entre sí y, por lo tanto, estarían dando cuenta de distintos aspectos de la percepción de desigualdad. Además, el estatus socioeconómico demostró influir directamente en la desigualdad percibida, mientras aquellos que evalúan su salario como justo tenderían a percibir menos desigualdad. Finalmente se discuten las consecuencias y proyecciones de esta investigación.
\end{abstract}

Palabras clave: desigualdad económica, indicadores, percepción, encuestas

Even though a series of perceived inequality indicators have been used in survey research, to date there is no research comparing these indicators. Using data from the 2009 Chilean ISSP national representative survey $(N=1,505)$, this research compared 3 measures of inequality perception and analyzed the influence of status, political position and evaluation of wage justice on perceptual variables, through correlation and regression methods. Results showed that the perceptual indicators are weakly associated with each other and, therefore, they can be regarded as covering different aspects of inequality perception. Furthermore, socioeconomic status had a direct influence on inequality perception, whereas those that evaluate their salary as fair tend to perceive less inequality. The consequences and projections of the study are addressed in the final discussion.

Keywords: economic inequality, indicators, perception, surveys

La desigualdad económica en Chile es un tema constante en los discursos públicos durante la última década. Uno de los principales aspectos que se releva en este ámbito es que Chile es uno de los países que lidera los rankings de desigualdad económica a nivel mundial. El índice de desigualdad de Gini lo ubica dentro de los 15 países con la peor distribución del ingreso a nivel internacional (United Nations Development Programme, 2010). La abundante evidencia de indicadores objetivos de desigualdad en el país contrasta con la escasa atención otorgada a aspectos subjetivos de la desigualdad, lo cual nos lleva a plantear la pregunta ¿En qué medida la desigualdad económica es percibida por la población? Tal pregunta adquiere relevancia, ya que la evidencia internacional señala una serie de posibles sesgos en la percepción de desigualdad, los que, finalmente, podrían repercutir en actitudes de tolerancia e incluso legitimación de situaciones de desigualdad. Nuestro argumento central es que para responder a esta pregunta

Juan Carlos Castillo, Daniel Miranda y Diego Carrasco, Centro de Medición MIDE UC, Escuela de Psicología, Pontificia Universidad Católica de Chile, Santiago, Chile.

Este trabajo fue realizado como parte del proyecto Inicio UC 24/2010 Testing the stability of perception and justification of economic inequality in Chile: A cross-sectional time comparison, financiado por la Vicerrectoría de Investigación de la Pontificia Universidad Católica de Chile.

La correspondencia relativa a este artículo debe ser dirigida a Juan Carlos Castillo, Centro de Medición MIDE UC, Escuela de Psicología, Pontificia Universidad Católica de Chile, Avda. Vicuña Mackenna 4860, Macul, Santiago, Chile. E-mail: jcastillov@uc.cl 
se requiere contar primero con indicadores específicos de percepción de desigualdad. Por ello, nuestro objetivo fue contribuir en esta línea mediante la comparación de distintos indicadores de desigualdad percibida y el análisis de la medida en que la posición que los individuos ocupan en la sociedad (i.e., estatus social) influye en la percepción de desigualdad. Aun cuando es posible identificar en Chile algunos estudios sobre actitudes y creencias sobre desigualdad (Garretón \& Cumsille, 2002; Lehmann \& Hinzpeter, 2000, 2001; Programa de Naciones Unidas para el Desarrollo, 1998), el análisis comparativo de indicadores de desigualdad percibida no tiene precedentes en la literatura revisada. Consideramos que realizar esta comparación en Chile otorga un valor especial al análisis aquí planteado, dada la alta concentración de ingresos existente en este país (Beyer \& Le Foulon, 2002; Hojman, 1996; Ruiz-Tagle, 1999; Torche, 2005).

El estudio de la percepción ocupa un lugar central en la psicología y, en particular, en la psicología social (Augoustinos, Walker \& Donaghue, 2006; Bodenhausen \& Lambert, 2003; Howard, 1994). La investigación en este ámbito se ha centrado en explicar diferencias individuales en términos de certezas y sesgos perceptuales, diferencias que han sido asociadas a una serie de factores internos o motivacionales, así como a factores situacionales, tales como los roles, la posición social o la identidad social (Levine, Resnick \& Higgins, 1993). Si bien la percepción de desigualdad económica no constituye un ámbito de estudio tradicional de la psicología social, actualmente se ha expresado preocupación por su inclusión como un objeto de estudio relevante, particularmente en el contexto de tender puentes (bridging) entre la psicología social y disciplinas afines, como la sociología (Fiske \& Molm, 2010; Melamed \& North, 2010). El presente artículo pretende avanzar en esta dirección, considerando a la percepción de desigualdad como objeto de cognición social situado en un contexto, explorando las variaciones individuales y posibles sesgos perceptuales en base a la posición que las personas ocupan en la sociedad, enmarcados en una aproximación sociológica a la psicología social (Cook, Fine \& House, 1995; Eagly \& Fine, 2010).

\section{La Percepción de la Desigualdad Económica}

Estudios sobre percepción de desigualdad económica han conducido a dos hallazgos relevantes que contrastan con posibles supuestos del sentido común. En primer lugar, si bien podríamos anticipar que en contextos con mayor desigualdad económica se percibe una mayor desigualdad, la evidencia internacional disponible sugiere que la desigualdad percibida dista de ser un "espejo" de la realidad, ya que sociedades con alta desigualdad no necesariamente expresan (en promedio) una alta desigualdad percibida (Castillo, Gerlitz \& Schrenker, 2008, Agosto; Castillo, Mühleck \& Wegener, 2008, Febrero). Un segundo supuesto corresponde a la creencia de que aquellos más afectados por situaciones de desigualdad, como las personas de menor estatus social, perciben mayor desigualdad. Sin embargo, investigaciones de finales de los noventa en el área de prestigio profesional revelan que la capacidad de discriminar entre salarios para ocupaciones de alto y bajo estatus disminuye de acuerdo al estatus individual (Wegener, 1987, 1990, 1992). Es decir, al menos en términos de percepción de desigualdad salarial, a menor estatus menor es la desigualdad percibida. En general, los resultados de investigaciones nos señalan que la percepción de desigualdad posee variaciones relevantes en la población, las que serían afectadas por una serie de determinantes contextuales e individuales. Además, la influencia de estos determinantes muchas veces adquiere un carácter contra intuitivo.

Las interrogantes en torno a la desigualdad percibida en la distribución de bienes se han abordado principalmente a partir de dos aproximaciones. La primera se enfoca en las creencias y preferencias respecto de la distribución económica, principalmente en la búsqueda de las reglas que legitiman o no la distribución del ingreso económico (Aalberg, 2003; Kluegel, Mason \& Wegener, 1995; Kluegel \& Smith, 1986; Ng \& Allen, 2005). La segunda aproximación se concentra en la percepción sobre la distribución económica sin el componente evaluativo, es decir, en qué medida las personas perciben el nivel de desigualdad económica existente en la 
sociedad. Hacemos esta distinción para relevar un aspecto que usualmente se confunde en la literatura, considerando que cuando se menciona la percepción sobre justicia distributiva en general se hace referencia a la evaluación sobre lo percibido como justo o injusto y no a la mera percepción sobre la desigualdad económica. Como mencionamos anteriormente, nos interesa posicionar este último punto de vista.

En investigaciones basadas en encuestas el indicador que tradicionalmente se utiliza para dar cuenta de la percepción de desigualdad es de carácter genérico. Este indicador consiste en un ítem medido en una escala Likert de acuerdo-desacuerdo respecto de la existencia de grandes desigualdades en la sociedad, el cual es frecuentemente incorporado en encuestas de opinión pública nacionales y comparativas. En términos de medición, es discutible que tal indicador pueda abarcar de manera comprehensiva un fenómeno complejo, como es la percepción de desigualdad. Para superar esta limitación proponemos incorporar al análisis de percepción de desigualdad dos indicadores adicionales, a saber, percepción de brechas salariales y percepción diagramática de desigualdad:

1. Brechas salariales. Consiste en una medida indirecta de percepción de desigualdad basada en respuestas sobre los salarios que se cree que obtienen ciertas ocupaciones. En concreto, se pregunta respecto del salario que el encuestado cree que obtiene alguien en una ocupación de alto estatus y alguien en una ocupación de bajo estatus. En base a estas dos magnitudes se calcula una proporción denominada brecha salarial percibida, dando como resultado una variable que puede ser considerada continua.

2. Percepción diagramática. Consiste en una evaluación gráfica sobre la distribución económica de una sociedad, línea de investigación que comienza en Hungría a fines de los años setenta (Evans, Kelley \& Kolosi, 1992). A partir de un set de imágenes (ver Figura 1), las personas deben responder qué diagrama representa mejor la distribución económica de su país.

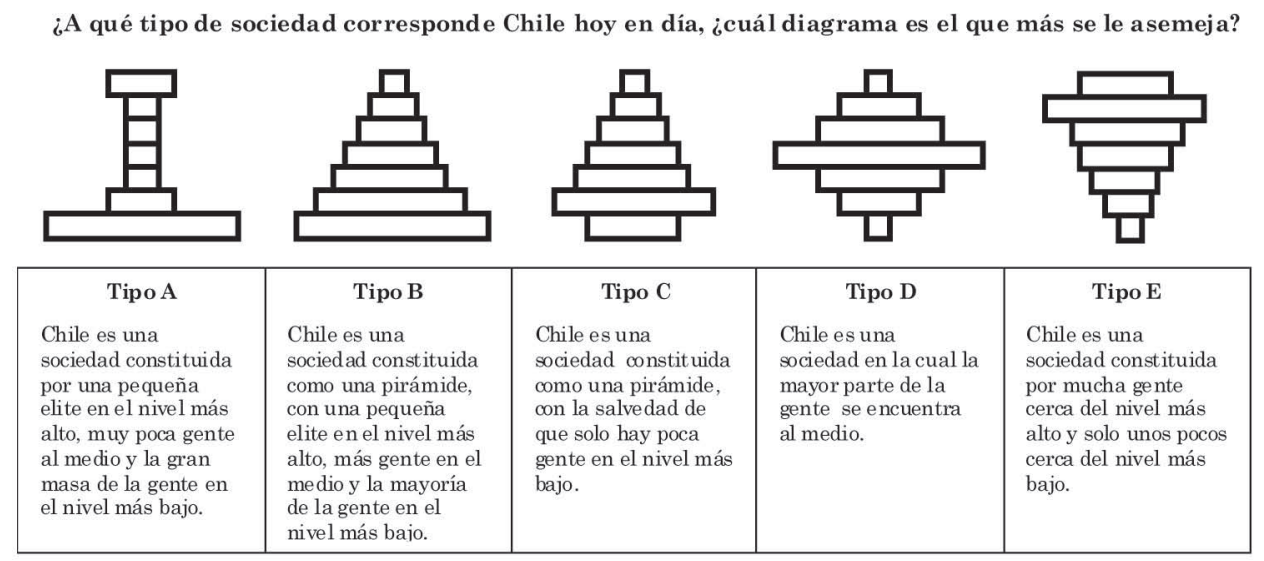

Figura 1. Ítem de percepción gráfica de distribución económica. Fuente: Encuesta ISSP 2009 (Centro de Estudios Públicos [CEP], 2009a).

El tipo A representa una pequeña elite en la parte superior y una gran masa en la parte inferior. El tipo B es una distribución tipo piramidal que disminuye gradualmente desde abajo hacia arriba en la escala social. El tipo C es similar a una pirámide, excepto que la gran masa no se acumula en el segmento más bajo. El tipo D presenta una acumulación en el centro, representando una gran clase media. Finalmente, en el tipo E gran parte de las personas se encuentra en los niveles superiores de la distribución. Por lo tanto, los diagramas pueden ser concebidos como imágenes que reflejan dónde se encuentra la mayor parte de la población en términos de estratificación, partiendo con una sociedad en la que la mayoría posee pocos re- 
cursos (A) y avanzando progresivamente hacia una sociedad en la que la mayoría posee más recursos (E). En este sentido, las opciones de respuesta dan como resultado una variable que puede ser tratada como ordinal. Entre las pocas referencias a análisis realizados en base a esta escala encontramos el estudio de Evans et al. (1992), el que sugiere que existen variaciones en los países según la cultura política (comparando Hungría y Australia): la cultura socialista influiría en la percepción de la sociedad marcadamente dividida entre clases (tipo A). Además, un estudio publicado por Cuneo (1996) con datos de Canadá muestra que el estatus social subjetivo actúa como un predictor de las distintas imágenes de la desigualdad.

Mediante análisis paralelos de las tres variables descritas — percepción general, brechas salariales y percepción diagramática - se espera dar cuenta de diferentes aspectos de la percepción sobre la desigualdad económica. Consideramos que las tres estarían entregando información de una misma dimensión, si bien desde diferentes ángulos. Por tanto, una primera hipótesis general de trabajo es que estas tres variables presentan una asociación significativa, ya que, a pesar de sus diferencias, se espera que abarquen aspectos de un mismo fenómeno.

\section{Determinantes de la Desigualdad Percibida}

En esta sección nos abocaremos a las diferencias individuales en percepción de desigualdad, las que serían susceptibles de ser explicadas mediante una serie de determinantes. Por lo tanto, nos referimos a un modelo conceptual de tipo explicativo que considera como variables dependientes a las tres medidas de percepción de desigualdad mencionadas, tal como se ilustra en la Figura 2.

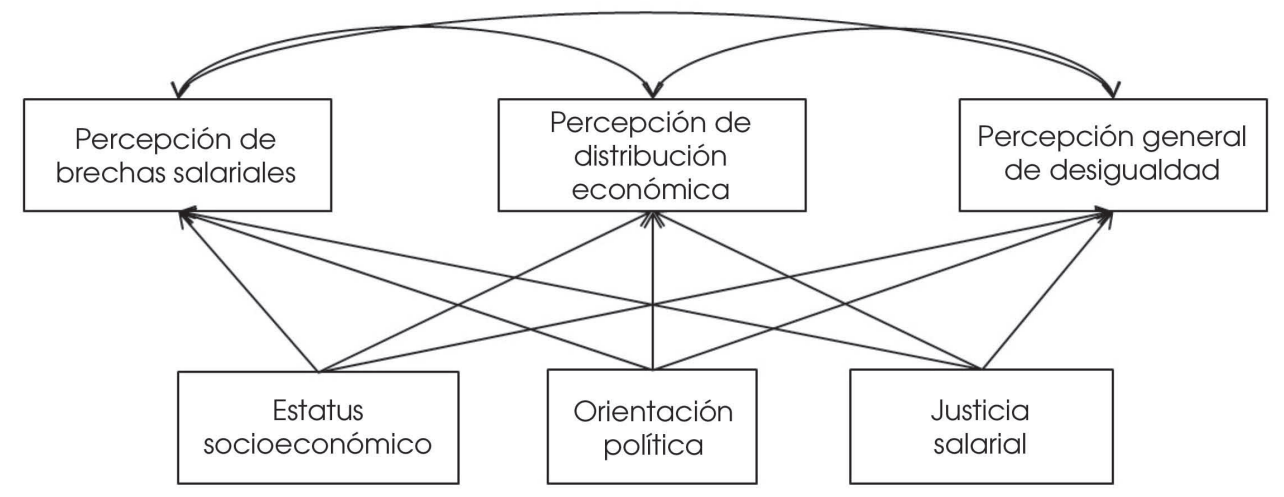

Figura 2. Modelo de percepción de desigualdad económica.

El modelo muestra en la parte superior de la Figura 2 las tres medidas de percepción de desigualdad asociadas entre sí, más tres determinantes en base a los cuales desarrollamos hipótesis de trabajo para explicar la variabilidad interindividual de percepciones de desigualdad: estatus socioeconómico, orientación política y justicia salarial.

\section{Estatus Socioeconómico}

Estudios revelan que aquellos individuos con mayor ingreso económico y nivel educacional poseen una capacidad mayor para distinguir entre el prestigio de una ocupación de alto estatus y el de una de bajo estatus (Wegener, 1987, 1990). La hipótesis propuesta es que a mayor estatus socioeconómico, mayor es la desigualdad percibida.

\section{Orientación Política}


Aun cuando no tenemos antecedentes específicos de una asociación entre orientación política y percepción de desigualdad, en la literatura existen referencias que relacionan posturas políticas con actitudes hacia la redistribución económica. En esta línea Gijsberts (1999) argumenta que "las personas que piensan que la distribución de ingreso debería hacerse más igualitaria (...) son más propensas a votar por un partido de izquierda que por un partido de derecha" (p. 111). Esta asociación ha sido también abordada en estudios de psicología política que vinculan el conservadurismo de derecha con una mayor tolerancia por la desigualdad económica (Jost, Glaser, Kruglanski \& Sulloway, 2003). Si bien en la presente investigación no abordamos preferencias respecto de la desigualdad sino percepciones, es posible proponer que el tema de desigualdad sería más saliente para aquellas personas que se identifican con la izquierda del espectro político y, por lo tanto, podrían expresar una mayor percepción de desigualdad económica, basadas en mayor información o a modo de denuncia.

\section{Justicia Salarial}

El tercer determinante de este estudio se refiere a la evaluación de justicia respecto del propio salario y su impacto en la desigualdad percibida. Teorías de justicia de ingresos, tales como la deprivación relativa (Crosby, 1979; Runciman, 1966) y la teoría de la equidad (Adams, 1963; Berkowitz \& Walster, 1976), señalan que la evaluación de justicia se basa en procesos de comparación social, es decir, mediante la comparación del propio salario con el salario que obtienen otras personas. En este marco la hipótesis sugerida es que aquellos que se sienten injustamente recompensados son más sensibles a las diferencias de ingresos y, por lo tanto, la comparación con individuos de salarios mayores llevará a que ellos extiendan el continuo de ingresos en términos perceptuales, percibiendo, por tanto, más desigualdad. Por otro lado, se espera que quienes se sienten más justamente recompensados minimicen las diferencias de ingreso, ya que el ser justamente recompensado en un contexto de alta desigualdad podría generar disonancia cognitiva (Festinger, 1957).

\section{Método}

\section{Participantes}

El International Social Survey Programme (ISSP) es un proyecto colaborativo de encuestas comparativas que cubren una amplia gama de temas sociales. Comenzó en 1983 con cuatro países fundadores (Alemania, Estados Unidos, Reino Unido y Australia) y en la actualidad participan más de 40. Cada año el cuestionario cubre un tema particular, el cual se repite en versiones sucesivas de la encuesta, permitiendo comparaciones transversales en el tiempo. La desigualdad social ha sido el tema de investigación de los módulos 1987, 1992, 1999 y 2009 del ISSP. En el presente estudio de carácter transversal empleamos los datos del año 2009 provistos por el Centro de Estudios Públicos correspondientes al Estudio Nacional de Opinión Pública $N^{\circ} 30$, Tercera Serie (CEP, 2009a), en el cual se encuentran incluidos los datos del ISSP 2009. La encuesta cuenta con 1.505 casos observados (58\% mujeres y $42 \%$ hombres mayores de 18 años, edad $M=46, S D=17)$.

El muestreo fue probabilístico por conglomerados, en primer lugar, por regiones del país y, en segundo lugar, según zona urbana y rural. En base a esa estratificación se seleccionó aleatoria y proporcionalmente a 301 unidades primarias de muestreo (manzanas) para cada región, provincia y comuna de Chile. En una segunda etapa se seleccionó, por muestreo sistemático, un total de cinco familias por manzana para, finalmente, seleccionar aleatoriamente al entrevistado. Este estudio consideró un margen de error de $\pm 2,7 \%$ con un nivel de confianza de un $95 \%$ (CEP, 2009a).

Los análisis incluyen los factores de expansión provistos en la base de datos de la encuesta. 


\section{Instrumento}

El cuestionario de la ISSP 2009 incluye una serie de ítems actitudinales acerca del ingreso, la distribución, la percepción de desigualdad, principios distributivos, expectativas acerca del rol del Estado sobre la desigualdad, así como preguntas acerca de la percepción y evaluación de las remuneraciones.

En este estudio la percepción general de desigualdad corresponde a la percepción general de distribución en Chile, mediante el grado de acuerdo o desacuerdo con la afirmación "Las diferencias de ingreso en Chile son demasiado grandes". La percepción de brechas salariales consiste en la proporción de salarios percibidos para una ocupación de alto estatus (el presidente de una gran empresa nacional) y una de bajo estatus (un obrero no calificado de una fábrica). Cuando esta proporción equivale a 1 , indica la ausencia de diferencias de brecha salarial percibida, aumentando mientras mayor distancia sea percibida entre ambos salarios. Para efectos de los análisis en los modelos de regresión esta variable la transformamos logarítmicamente. Finalmente, el indicador de percepción diagramática de distribución económica consta de cinco esquemas que expresan distribuciones posibles del país. En la Tabla 1 presentamos la descripción de las variables dependientes.

Tabla 1

Variables Dependientes

\begin{tabular}{|c|c|c|c|}
\hline Variable & Ítem & Respuesta & Descriptivos \\
\hline $\begin{array}{l}\text { Percepción } \\
\text { general de } \\
\text { desigualdad }\end{array}$ & $\begin{array}{l}\text { Las diferencias de } \\
\text { ingreso en Chile son } \\
\text { demasiado grandes. }\end{array}$ & $\begin{array}{l}\text { 1. Muy en desacuerdo } \\
\text { 2. En desacuerdo } \\
\text { 3. Ni de acuerdo ni en desacuerdo } \\
\text { 4. De acuerdo } \\
\text { 5. Muy de acuerdo }\end{array}$ & $\begin{array}{lr}\text { 1) } & 1,07 \% \\
\text { 2) } & 4,36 \% \\
\text { 3) } & 7,04 \% \\
\text { 4) } & 49,73 \% \\
\text { 5) } & 37,80 \%\end{array}$ \\
\hline $\begin{array}{l}\text { Brecha } \\
\text { salarial }\end{array}$ & $\begin{array}{l}\text { ¿Cuánto cree Ud. que } \\
\text { gana al mes } \\
\ldots \text { un obrero no calificado } \\
\text { de una fábrica? } \\
\text {... el presidente de una } \\
\text { gran empresa nacional? }\end{array}$ & Brecha $=\ln (\$$ Gerente $/ \$ O b r e r o)$ & $\begin{array}{l}\text { Media }=61,87 \\
D E=97,33 \\
\text { Mediana }=32,00\end{array}$ \\
\hline $\begin{array}{l}\text { Percepción } \\
\text { diagramática } \\
\text { de distribución }\end{array}$ & $\begin{array}{l}\text { ¿Qué tipo de sociedad } \\
\text { es Chile hoy? ¿A cuál } \\
\text { diagrama se acerca más? }\end{array}$ & 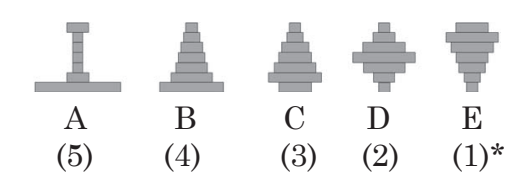 & $\begin{array}{l}\text { 1) } 2,89 \% \\
\text { 2) } 11,68 \% \\
\text { 3) } 13,13 \% \\
\text { 4) } 47,97 \% \\
\text { 5) } 24,33 \%\end{array}$ \\
\hline
\end{tabular}

\footnotetext{
* Los diagramas se codifican en el sentido que 1 representa menor percepción de desigualdad y 5 mayor percepción de desigualdad.
}

Las variables independientes se componen de las variables estatus (ingreso y educación), posición política y justicia del propio salario. El ingreso lo operacionalizamos como el ingreso neto equivalente, obtenido de la división del ingreso familiar mensual por el número de personas que comparten el mismo hogar. Educación, por su parte, corresponde al último nivel educacional alcanzado por el participante. En relación a la posición política, utilizamos una escala de identificación o simpatía con sectores políticos desde izquierda a derecha. Finalmente, la justicia del propio salario corresponde a un ítem del cuestionario en escala Likert que pregunta 
al entrevistado si considera justa su remuneración. La descripción de estas variables la podemos ver en la Tabla 2.

Tabla 2

Variables Independientes

\begin{tabular}{|c|c|c|c|}
\hline Dimensión & Variable & Operacionalización & Descriptivos \\
\hline & Estatus subjetivo & 1 (Bajo) a 10 (Alto) & $\begin{array}{l}\text { Media }=4,03 \\
D E=1,66 \\
\text { Mediana }=4,00\end{array}$ \\
\hline & $\begin{array}{l}\text { Ingreso neto } \\
\text { equivalente }\end{array}$ & $\begin{array}{l}\text { Ingreso del hogar / cantidad de } \\
\text { personas que componen el hogar }\end{array}$ & $\begin{array}{l}\text { Media }=11,31 \\
D E=0,97 \\
\text { Mediana }=11,28\end{array}$ \\
\hline Estatus & Nivel educacional & $\begin{array}{l}\text { Educación básica incompleta } \\
\text { Educación básica completa } \\
\text { Educación media incompleta } \\
\text { Educación media completa } \\
\text { Educación universitaria incompleta } \\
\text { Educación universitaria completa }\end{array}$ & $\begin{array}{l}22,04 \% \\
12,69 \% \\
13,09 \% \\
24,58 \% \\
11,42 \% \\
16,17 \%\end{array}$ \\
\hline Posición política & $\begin{array}{l}\text { Escala de simpatía/ } \\
\text { preferencia por } \\
\text { sector político }\end{array}$ & $\begin{array}{l}\text { Derecha } \\
\text { Centro } \\
\text { Izquierda } \\
\text { Ninguna }\end{array}$ & $\begin{array}{l}21,26 \% \\
13,43 \% \\
22,65 \% \\
42,66 \%\end{array}$ \\
\hline Justicia salarial & $\begin{array}{l}\text { Justicia del propio } \\
\text { salario }\end{array}$ & $\begin{array}{l}\text { ¿Es justa su remuneración? } \\
\text { 1. Mucho menos de lo que es justo } \\
\text { 2. Un poco menos de lo que es justo } \\
\text { 3. Lo que es justo para mí } \\
\text { 4. Un poco más de lo que es justo } \\
\text { 5. Mucho más de lo que es justo }\end{array}$ & $\begin{array}{lr}\text { 1) } & 31,86 \% \\
\text { 2) } & 39,07 \% \\
\text { 3) } 24,43 \% \\
\text { 4) } 3,46 \% \\
\text { 5) } 1,18 \%\end{array}$ \\
\hline
\end{tabular}

\section{Procedimiento}

El procedimiento de recolección de datos se realizó mediante entrevistas personales cara a cara con una duración aproximada de una hora. Las entrevistas se realizaron en los hogares de los entrevistados y fue voluntaria. Hubo un $1,6 \%$ de reemplazo de individuos por rechazo a responder el cuestionario (CEP, 2009b).

\section{Análisis de Datos}

Para el análisis contemplamos, en primer lugar, resultados de tipo descriptivo, seguidos de correlaciones policóricas y poliseriales y, finalmente, modelos de regresión. El tipo de regresión lo ajustamos al nivel de medición de cada una de las variables dependientes: regresión OLS para el caso de la brecha (variable continua) y logística ordinal para el caso de la percepción de distribución económica y percepción general de desigualdad (variables categóricas). 


\section{Resultados}

\section{Análisis Descriptivo}

Respecto de la percepción general de desigualdad, observamos un alto grado de acuerdo en la afirmación "Las diferencias de ingreso en Chile son demasiado grandes" (Tabla 1), sobre la que más del 87\% de las personas plantea estar de acuerdo o muy de acuerdo, lo cual es concordante con publicaciones anteriores (Comisión Económica para América Latina [CEPAL], 2010). Este aparente consenso perceptual contrasta con la variabilidad que observamos en las respuestas de percepción de salarios. En la Figura 3 aparece un gráfico de cajas sobre la percepción de ingreso para cargos de alto estatus y bajo estatus, el cual permite hacerse una idea de la distribución de las respuestas en términos de tendencia central y variabilidad.

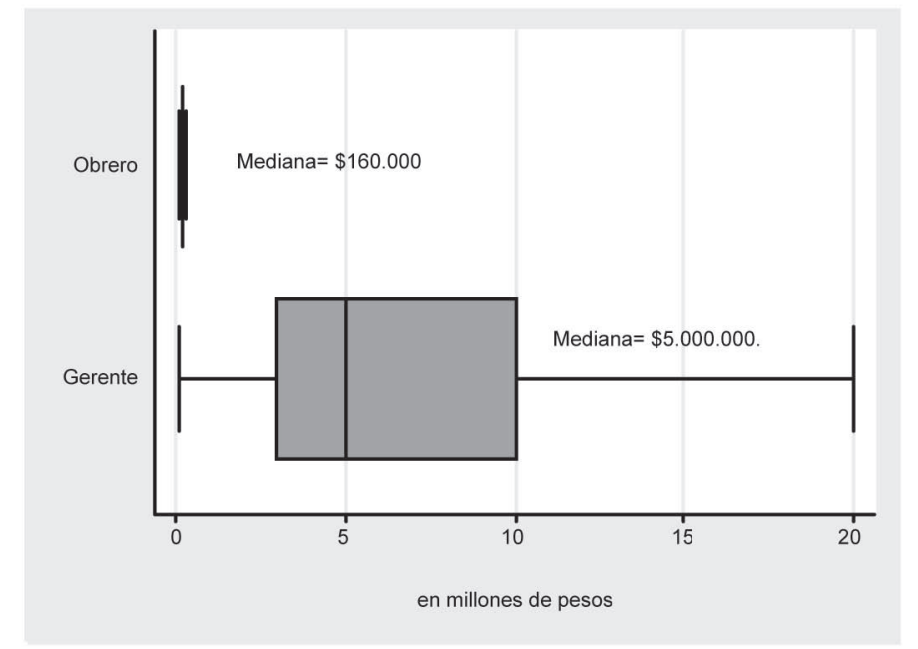

Figura 3. Mediana y distribución de los salarios mensuales percibidos para gerente y obrero. Fuente: Encuesta ISSP 2009 (CEP, 2009a).

Al comparar ambas ocupaciones, el salario percibido para el cargo de gerente es claramente más alto que el ingreso del obrero. También existe un mayor acuerdo entre los entrevistados respecto de cuánto gana en el país una ocupación de bajo estatus, concordante con la evidencia internacional (Kelley \& Evans, 1993). La menor variabilidad de este salario podría estar influida por la información que existe respecto del salario mínimo en el país (que se aproxima bastante a la mediana percibida para el obrero). El cargo de alto estatus no cuenta con referentes precisos, lo que se refleja en una alta dispersión en torno a la mediana. Por lo tanto, las diferencias que se presenten en la brecha salarial percibida estarán mayormente relacionadas con el salario de la ocupación de alto estatus. Con todo, la brecha de percepción de ingresos sería de 31,25, es decir, se percibe que aquellas personas que ocupan cargos de alto estatus ganan alrededor de 31 veces más que aquellas personas que ocupan cargos de bajo estatus. 
En cuanto a los diagramas que representan la distribución en la sociedad, el $72 \%$ de las personas se ubica en los diagramas tipos A y B, los que se asocian a una mayor percepción de desigualdad en la distribución de bienes (ver Tabla 1), ya que en estos dos diagramas la mayor parte de la población se encuentra representada en los niveles más bajos de la estructura social. Por lo tanto, al igual que en la percepción general de desigualdad, este indicador nos señala que existe un gran acuerdo respecto de la desigualdad en Chile, lo que también se ha observado previamente en países como Brasil, Portugal, Rusia (Scalon, 2009), Hungría, Polonia y Bulgaria (Cuneo, 1996).

El análisis de correlaciones entre los tres indicadores que mostramos en la Tabla 3 no genera evidencia suficiente para sustentar la hipótesis de asociación entre ellos, ya que la única correlación significativa es entre la brecha percibida y la percepción general, si bien el grado de asociación es muy bajo, $r(1502)=0,09, p<0,01$. Tal hallazgo nos sugiere en principio que los tres indicadores miden distintos aspectos de la desigualdad percibida y, en particular, nos permite señalar que la percepción diagramática no se encuentra asociada a aspectos específicos de la desigualdad en términos salariales. Como los indicadores constituyen medidas de ítem único, no existe posibilidad de calcular la confiabilidad por consistencia interna y los indicadores se exponen, además, a tener una baja estabilidad temporal.

Tabla 3

Correlación Entre Indicadores de Percepción de Desigualdad Económica

\begin{tabular}{lccc}
\hline & $\begin{array}{c}\text { Percepción } \\
\text { general }\end{array}$ & $\begin{array}{c}\text { Brecha } \\
\text { percibida }\end{array}$ & $\begin{array}{c}\text { Percepción } \\
\text { diagramática }\end{array}$ \\
\hline $\begin{array}{l}\text { Percepción } \\
\text { general }\end{array}$ & 1,00 & & \\
$\begin{array}{l}\text { Brecha } \\
\text { percibida }\end{array}$ & $0,09 * *$ & 1,00 & \\
$\begin{array}{l}\text { Percepción } \\
\text { distribución }\end{array}$ & 0,04 & $0,00^{\text {a }}$ & 1,00 \\
$\begin{array}{l}\text { Notas. Correlaciones poliseriales, excepto "a" que corresponde al coeficiente de } \\
\text { correlación policórica. } \\
\text { ** } p<0,01, * p<0,05 . \\
\text { Entre paréntesis: valores } z .\end{array}$ & &
\end{tabular}

\section{Análisis Bivariado de la Influencia de los Predictores en la Desigualdad Percibida}

En esta fase del análisis descriptivo entregamos antecedentes respecto de la asociación de cada una de las variables independientes con cada una de las variables dependientes, información que resumimos en la Tabla 4. 
Tabla 4

Puntajes Promedio de Percepción de Desigualdad Según Variables de Estatus, Posición Política y Justicia Salarial

\begin{tabular}{|c|c|c|c|}
\hline Variables & $\begin{array}{l}\text { Percepción } \\
\text { general }\end{array}$ & $\begin{array}{l}\text { Brecha } \\
\text { percibida }\end{array}$ & $\begin{array}{l}\text { Percepción } \\
\text { diagramática }\end{array}$ \\
\hline \multicolumn{4}{|l|}{ Quintil de ingreso } \\
\hline Quintil 1 & 4,2 & 31,3 & 3,9 \\
\hline Quintil 2 & 4,2 & 27,8 & 3,9 \\
\hline Quintil 3 & 4,2 & 33,3 & 3,8 \\
\hline Quintil 4 & 4,2 & 36,0 & 3,7 \\
\hline \multirow[t]{2}{*}{ Quintil 5} & 4,2 & 44,4 & 3,4 \\
\hline & $\mathrm{Rho}^{\mathrm{a}}=0,01, p=0,820$ & $\mathrm{Rho}^{\mathrm{b}}=0,09, p=0,005$ & $\mathrm{Rho}^{\mathrm{a}}=0,16, p=0,001$ \\
\hline \multicolumn{4}{|l|}{ Nivel educacional } \\
\hline Ed. básica incompleta & 4,1 & 25,2 & 4,0 \\
\hline Ed. básica completa & 4,1 & 27,8 & 3,9 \\
\hline Ed. media incompleta & 4,2 & 31,3 & 3,9 \\
\hline Ed. media completa & 4,2 & 30,0 & 3,8 \\
\hline Ed. superior no universitaria & 4,2 & 35,7 & 3,7 \\
\hline \multirow[t]{2}{*}{ Ed. universitaria } & 4,3 & 47,1 & 3,6 \\
\hline & $\mathrm{Rho}^{\mathrm{a}}=0,08, p=0,006$ & $\mathrm{Rho}^{\mathrm{b}}=0,13, p=0,001$ & $\mathrm{Rho}^{\mathrm{a}}=0,13, p=0,001$ \\
\hline \multicolumn{4}{|l|}{ Estatus subjetivo } \\
\hline Bajo & 4,2 & 26,1 & 4,0 \\
\hline Medio bajo & 4,2 & 35,7 & 3,9 \\
\hline Medio & 4,2 & 32,3 & 3,6 \\
\hline Medio alto & 4,2 & 32,0 & 3,4 \\
\hline \multirow[t]{2}{*}{ Alto } & 3,9 & 25,1 & 3,3 \\
\hline & $\mathrm{Rho}^{\mathrm{a}}=-0,05, p=0,096$ & $\mathrm{Rho}^{\mathrm{b}}=0,01, p=0,750$ & $\mathrm{Rho}^{\mathrm{a}}=0,18, p=0,001$ \\
\hline \multicolumn{4}{|l|}{ Posición política } \\
\hline Derecha & 4,2 & 35,7 & 3,7 \\
\hline Centro & 4,1 & 35,7 & 3,8 \\
\hline Izquierda & 4,2 & 35,0 & 3,7 \\
\hline \multirow[t]{2}{*}{ Ninguna } & 4,2 & 27,8 & 3,8 \\
\hline & $\begin{array}{c}\chi^{2}(12, N=1431)= \\
18,91, p=0,09\end{array}$ & $\begin{array}{c}\mathrm{F}(3,1275)=0,49 \\
p=0,69\end{array}$ & $\begin{array}{c}\chi^{2}(12, N=1398)= \\
16,78, p=0,16\end{array}$ \\
\hline \multicolumn{4}{|l|}{ Percepción de salario propio } \\
\hline Mucho menos de lo que es justo & 4,3 & 31,4 & 4,0 \\
\hline Un poco menos de lo que es justo & 4,2 & 32,4 & 3,8 \\
\hline Lo que es justo para mí & 4,0 & 33,3 & 3,6 \\
\hline Un poco más de lo que es justo & 4,1 & 33,3 & 3,5 \\
\hline \multirow[t]{2}{*}{ Mucho más de lo que es justo } & 4,0 & 21,4 & 3,8 \\
\hline & $\mathrm{Rho}^{\mathrm{a}}=-0,14, p=0,001$ & $\mathrm{Rho}^{\mathrm{b}}=0,00, p=0,992$ & $\mathrm{Rho}^{\mathrm{a}}=0,14, p=0,001$ \\
\hline
\end{tabular}

Notas: Para las variables Percepción general y Percepción de distribución el dato que reportamos es la mediana; para la variable Brecha percibida, la media. Las correlaciones que reportamos son policóricas ${ }^{\mathrm{a}} \mathrm{y}$ poliseriales ${ }^{\mathrm{b}}$. Solo para posición política se especifica $\chi^{2}$ por ser una variable nominal. 
Se realizó un contraste lineal, cuadrático y cúbico entre estatus subjetivo y brecha percibida, no resultando significativo en ninguno de los casos: $F_{\text {lineal }}(1,1314)=0,72, p=0,39 ; F_{\text {cuadrático }}(1$, $1314)=0,93, p=0,33 ; F_{\text {cúbico }}(1,1314)=0,00, p=0,98$. El mismo contraste se realizó entre percepción de salario propio y brecha percibida, no resultando significativo en ninguno de los casos: $F_{\text {lineal }}(1,1198)=2,16, p=0,14 ; F_{\text {cuadrático }}(1,1198)=1,63, p=0,20 ; F_{\text {cúbico }}(1,1198)=0,04, p=0,84$.

Observamos que las variables de estatus (ingreso, educación y estatus subjetivo) no presentan asociación significativa con la percepción general de desigualdad. Por otro lado, y en concordancia con evidencia previa (Castillo, 2009; Wegener, 1987), apreciamos una asociación directa con la brecha salarial, lo que se refleja en que las personas clasificadas en el quintil de más bajos ingresos (brecha $=31,3$ ) perciben una brecha 13 veces menor que los del quintil 5 (brecha $=44,4$ ). En cuanto al estatus subjetivo, es relevante considerar que la ausencia de una asociación significativa con la brecha percibida puede relacionarse con la escasa variabilidad de este predictor $(M=4,02, D E=1,66)$, lo que nos señala que en Chile gran parte de la población piensa que es de clase media (aproximadamente el 65\% de la población se clasifica entre los niveles 3 y 5 en la escala de 1 a 10). En contraste, observamos una asociación inversa de las tres variables de estatus con la percepción diagramática, indicando que aquellas personas que se ubican más alto en la sociedad tienden a percibir a esta como menos desigual.

Respecto de la posición política, no observamos asociaciones significativas con ninguna de las tres variables dependientes, mientras que en la percepción de justicia salarial observamos que quienes consideran su salario como justo o más de lo justo tienden a una menor percepción general de desigualdad. Por último, en cuanto a percepción diagramática observamos una asociación inversa, lo que indica que aquellos que se sienten más injustamente recompensados tienden a percibir más desigualdad.

Hasta este punto del análisis podemos concluir que, exceptuando la posición política, las variables explicativas muestran distintos grados de asociación con las variables perceptuales. En la sección siguiente estimaremos la estabilidad de estas asociaciones bajo la influencia simultánea de predictores y controles sociodemográficos, mediante modelos de regresión.

\section{Modelos Explicativos de Percepción de Desigualdad}

En esta sección presentamos una serie de modelos que incluyen estatus, posición política y justicia salarial como predictores de las variables perceptuales. Presentamos los resultados de la estimación en la Tabla 5, en la que aparecen cinco modelos para cada variable dependiente, según la incorporación progresiva de predictores en una lógica paso-a-paso (stepwise). 


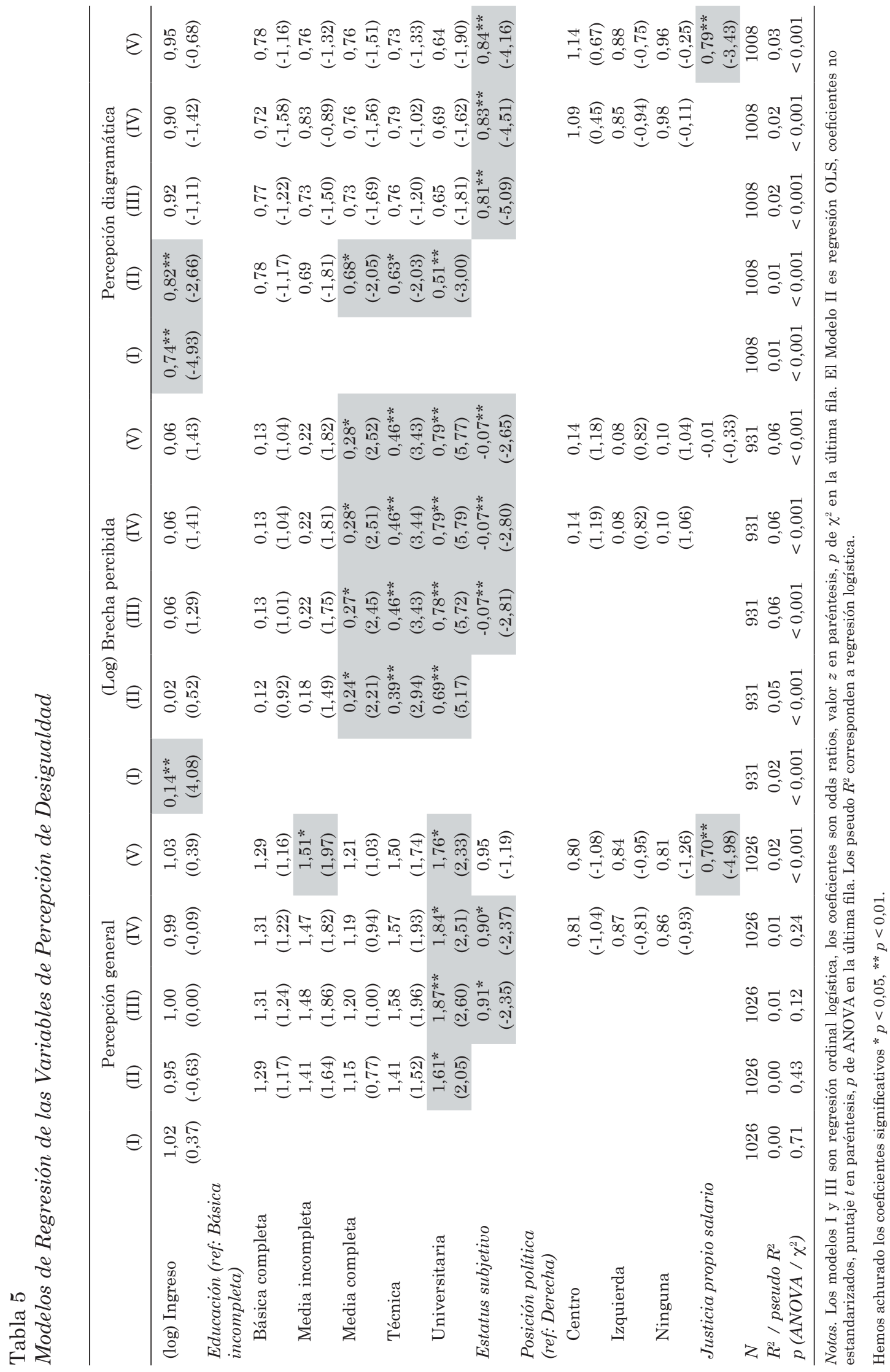


Respecto de las variables de estatus, vemos que el ingreso posee efectos significativos tanto en la brecha salarial como en la percepción diagramática, aun cuando de signo opuesto. Sin embargo, al ingresar predictores adicionales, este efecto se anula ante la presencia de variables educacionales, en el caso de la brecha, y del estatus subjetivo, en el caso de los diagramas. Por lo tanto, si bien el ingreso por si solo da cuenta de ciertas diferencias en percepción de desigualdad, no aparece como una variable decidora en presencia de otros predictores. La influencia del nivel educacional en la brecha percibida puede deberse a que a mayor nivel educacional existe un mayor acceso a información sobre salarios de alto estatus, elemento determinante de una mayor o menor brecha salarial percibida. Esta misma situación puede relacionarse con los efectos significativos de educación en la percepción general de desigualdad, si bien en este caso no se aprecia una tendencia clara a medida que aumenta el nivel educacional, sino más bien una diferenciación de todos los niveles respecto del nivel más alto (universitario), quienes tienden a percibir mayor desigualdad.

Una imagen distinta nos presenta la variable percepción diagramática de distribución económica en relación a variables de estatus. En este caso, los efectos individuales de ingreso y educación se anulan bajo el control de la variable estatus subjetivo. De acuerdo a estos resultados, es dable pensar que para evaluar la percepción de distribución económica lo relevante es saber dónde el entrevistado piensa que se encuentra en la escala social más que los antecedentes objetivos de estatus. El efecto del estatus subjetivo nos dice que los entrevistados tienden a asociar su estatus con el segmento mayoritario del país, ya que este segmento va subiendo de forma progresiva en los diagramas que se presentan en este ítem y, por tanto, se produce esta asociación inversa entre ambas variables (bajo estatus subjetivo - alta percepción de desigualdad). Dadas las características distribucionales del estatus subjetivo (que analizamos en la sección anterior), podemos decir que además de la creencia expandida de pertenecer a los segmentos medios, los chilenos también tienden a creer que pertenecen a la mayoría. Con todo, en el análisis conjunto de las influencia de variables de estatus encontramos evidencia parcial a favor de la hipótesis que plantea que la percepción de desigualdad tiende a variar positivamente con el estatus social (Wegener, 1987, 1990), particularmente con el nivel educacional.

La posición política corresponde a una segunda categoría de predictores que, como vemos en la Tabla 5, se caracteriza por su ausencia de efectos significativos en las tres variables perceptuales y confirma la tendencia observada en la sección bivariada, contradiciendo posturas clásicas de teorías marxistas respecto de la influencia de variables de tipo ideológico en elementos perceptuales en relación a la desigualdad (Abercrombie, Hill \& Turner, 1980; Huber \& Form, 1973). Ahora bien, es importante señalar que estamos hablando de percepciones y no de preferencias respecto a la distribución, ámbito en el cual sí encontramos evidencia en la literatura (Mühleck, 2009).

Por último, los modelos de regresión incorporan como predictor a la evaluación de justica del propio salario. Nuevamente apreciamos una influencia diferencial en las variables dependientes. El efecto inverso en la percepción general de desigualdad es el más comprensible desde un punto de vista racional: considero mi salario injusto, lo cual me lleva a manifestar una voz de protesta respecto de la distribución económica en el país. Es decir, se podría argumentar que la desigualdad se vuelve un tema saliente ante la evaluación de injusticia respecto del propio salario. Dado este efecto, llama la atención que esta evaluación de justicia/injustica no se manifieste en la brecha percibida, lo cual en términos teóricos contradice teorías clásicas de justicia distributiva que se basan en procesos de comparación social — tales como la teoría de la equidad (Adams, 1963; Berkowitz \& Walster, 1976)—, en los que la sensación de injusticia surge de la comparación del propio salario con los salarios de terceros, lo que llevaría a percibir una mayor brecha salarial. Por supuesto, acá surgen problemas de dirección de causalidad en la interpretación que, ante ausencia de datos experimentales o longitudinales, podemos solo intentar fundamentar teóricamente. Sin embargo, podríamos proponer que la injusticia del salario propio, si bien lleva a expresar descontento respecto de la distribución general, no se relaciona con información específica del medio respecto a desigualdad de ingresos en ámbitos 
como los salarios. Respecto de la percepción diagramática, el efecto inverso de la justicia salarial podemos vincularlo a argumentos anteriores en términos de consonancia cognitiva, ya que en la medida que considero mi salario justo, la imagen de una distribución desproporcionada aparece como disonante con la evaluación de justicia personal. Es decir, se produciría una tensión entre los ideales de justicia e igualdad que sería resuelta alterando la imagen que se posee de la sociedad: mi salario es justo, por lo tanto, la distribución económica no es tan desigual.

\section{Conclusiones}

Este estudio estuvo centrado en dos aspectos relacionados con la medición de percepción de desigualdad: diferencias entre indicadores y diferencias entre la influencia de predictores sobre estos indicadores. El análisis se enmarcó en el área de la psicología social sociológica, que se caracteriza por el énfasis en la influencia de variables de la estructura social en elementos de carácter cognitivo.

Como lección general podemos decir que la percepción de desigualdad es un fenómeno complejo que ha sido tratado hasta ahora de una forma simple. El indicador por excelencia de percepción de desigualdad en encuestas sociales es la percepción general de desigualdad, en base al cual se elaboran una serie de conclusiones que muchas veces se basan meramente en comparación de promedios generales por país. De nuestros análisis concluimos que este indicador no solo da cuenta de un aspecto parcial de la percepción de desigualdad, sino también de una escasa varianza y, por tanto, presenta limitaciones para avanzar en el estudio de los determinantes de este fenómeno. Por lo tanto, nuestra sugerencia es a ser más cauto cuando se habla de percepción de desigualdad y acompañar este indicador con medidas alternativas.

Nuestros resultados revelan que la desigualdad es percibida de forma desigual. Las imágenes de la sociedad distan de ser un "espejo" de la realidad, ya que se ven afectadas por una serie de variables que actúan como filtro o sesgo perceptual, lo que está en línea con investigaciones en el área de la cognición social que mencionamos al inicio del artículo. Estos filtros producen efectos que se alejan de lo esperable desde el sentido común, tales como la tendencia a percibir mayor desigualdad por parte de individuos de mayor estatus (particularmente mayor educación), la tendencia a sobre identificarse con estratos medios y el impacto de un mayor estatus subjetivo en una menor percepción de brechas. La relevancia de estos procesos de carácter socio-cognitivo va más allá del plano meramente académico, ya que es esperable que tengan consecuencias en acciones concretas de los individuos respecto de la distribución económica del país. Por ejemplo, si aquellos individuos de menor estatus perciben menos desigualdad, no es esperable que exista presión por instalar este tema en la agenda pública como un problema social relevante ni tampoco esperar cambios a corto plazo en términos redistributivos en el país. Estas posibles implicancias otorgan aún mayor relevancia al estudio de la percepción de la desigualdad en una realidad particular como Chile, uno de los países con mayor desigualdad económica a nivel mundial.

De nuestros resultados llama la atención, en primer lugar, la baja asociación entre percepción general de desigualdad y percepción de brechas salariales, ya que el salario es una de las fuentes principales de ingreso económico y, por lo tanto, se hubiera esperado una asociación más estrecha con una apreciación general respecto de la desigualdad. En otras palabras, la evaluación general de desigualdad económica parece no estar vinculada de forma prioritaria a desigualdades salariales en el país. En segundo lugar, también es llamativo el efecto inverso del estatus subjetivo en la brecha salarial percibida. Si bien esto va en contra de nuestras hipótesis, podemos avanzar algunas propuestas de manera exploratoria y que pueden ser tema de futuras investigaciones. En primer lugar, tenemos que considerar que estos modelos ya están bajo control de variables objetivas como educación e ingreso, es decir, lo que el estatus subjetivo nos indica en este caso es qué tanto influye el sesgo del estatus subjetivo respecto del objetivo en la percepción de brechas salariales. Por lo tanto, el efecto inverso del estatus 
subjetivo sugiere que aquellas personas que sobre evalúan su posición social tienden a percibir una menor brecha salarial. Este ajuste cognitivo, que bien puede ser asociado a teorías clásicas de disonancia cognitiva (Festinger, 1957; Jost, Pelham, Sheldon \& Sullivan, 2003), nos señala que aquellos de estatus bajo que creen pertenecer a un estatus superior requieren "acortar" el continuo de estatus. O sea, si yo soy de clase media, mi actual estatus no debería diferir tanto de una persona de alto estatus, así como tampoco mi salario de su salario. Tal operación, sin duda, provoca una distorsión respecto de la distribución económica que anteriormente ha sido vinculada a procesos de legitimación de desigualdad (Della Fave, 1980).

El vínculo entre percepción de desigualdad y formas de acción social es un primer elemento a mencionar como campo de futuras investigaciones. Si bien la acción social es difícil de operacionalizar en encuestas de opinión, existe la posibilidad de establecer indicadores en temas de participación política convencional y no convencional, los que esperamos poder incorporar en futuras encuestas. Un segundo aspecto a explorar son las percepciones en distintas dimensiones de la desigualdad, que van más allá de lo salarial y que abarcan ámbitos como la educación, salud y vivienda, entre otros. En tercer lugar, sugerimos la incorporación de elementos ideológicos de tipo distributivo como posibles determinantes de las percepciones de desigualdad y que vayan más allá de la posición política. Por ejemplo, es probable que individuos que se caractericen por preferencias redistributivas desde el Estado tiendan a percibir mayor desigualdad. Un cuarto aspecto se refiere a profundizar en las consecuencias de la evaluación de justicia respecto del propio salario, para lo que se recomienda que, además del ítem general utilizado en esta investigación, se incluyan preguntas específicas respecto del salario actual y el salario que se consideraría justo.

Finalmente, las limitaciones metodológicas de este diseño transversal en términos de modelos explicativos podrían ser compensadas mediante el análisis de datos de tipo longitudinal, así como también comparando los datos de Chile con los de otros países.

\section{Referencias}

Aalberg, T. (2003). Achieving justice: Comparative public opinion on income distribution. Leiden, Holanda: Brill. Abercrombie, N., Hill, S. \& Turner, B. (1980). The dominant ideology thesis. London, Reino Unido: Allen \& Unwin.

Adams, J. (1963). Towards an understanding of inequity. Journal of Abnormal Psychology, 67, 422-436. doi:10.1037/ h0040968

Augoustinos, M., Walker, I. \& Donaghue, N. (2006). Social cognition: An integrated introduction. London, Reino Unido: Sage.

Berkowitz, L. \& Walster, E. (Eds.) (1976). Advances in experimental social psychology, Vol. 9, Equity theory: Toward a general theory of social interaction. San Diego, CA: Academic Press.

Beyer, H. \& Le Foulon, C. (2002). Un recorrido por las desigualdades salariales en Chile. Estudios Públicos, 85, 139175.

Bodenhausen, G. V. \& Lambert, A. J. (Eds.) (2003). Foundations of social cognition: A festschrift in honor of Robert S. Wyer, Jr. Mahwah, NJ: Lawrence Erlbaum.

Castillo, J. (2009, Abril). ¿Consenso o conflicto acerca de la distribución? La legitimación de la desigualdad económica en Chile en perspectiva comparada. Ponencia presentada en el $2^{\text {nd }}$ Latin American Conference of the World Association for Public Opinion Research, Lima, Perú.

Castillo, J., Gerlitz, J. \& Schrenker, M. (2008, Agosto). Perception and legitimacy of income inequality in international comparison. Ponencia presentada en el APSA Annual Conference, Boston, MA, Estados Unidos.

Castillo, J., Mühleck, K. \& Wegener, B. (2008, Febrero). The empirical approach to the study of social justice: A research agenda for Latin America. Ponencia presentada en la Conference Intra- and Inter-Generational Transmission of Inequality, Ciudad de México, México.

Centro de Estudios Públicos (2009a). Estudio Nacional de Opinión Pública, Tercera Serie: manual del usuario Encuesta CEP N 30. Santiago, Chile: Autor.

Centro de Estudios Públicos (2009b). Estudio Nacional de Opinión Pública No 30, Tercera Serie: Documento de Trabajo $N^{o}$ 378. Santiago, Chile: Autor. Extraído de http://www.cepchile.cl/dms/lang_1/doc_4397.html

Comisión Económica para América Latina (2010). América Latina frente al espejo: dimensiones objetivas y subjetivas de la inequidad social y el bienestar en la región. Santiago, Chile: Autor.

Cook, K. S., Fine, G. A. \& House, J. S. (Eds.) (1995). Sociological perspectives on social psychology. Boston, MA: Allyn and Bacon.

Crosby, F. (1979). Relative deprivation revisited: A response to Miller, Bolce, and Halligan. American Political Science Review, 73, 103-112. doi:10.2307/1954733 
Cuneo, C. (1996). International images of social inequality: A ten-country comparison. En A. S. Frizzell \& J. H. Pammett (Eds.), Social inequality in Canada (pp. 31-65). Toronto, Canadá: Carleton University Press.

Della Fave, R. (1980). The meek shall not inherit the earth: Self evaluation and the legitimacy of stratification. American Sociological Review, 45, 955-971.

Eagly, A. \& Fine, G. A. (2010). Bridging social psychologies: An introduction. Social Psychology Quarterly, 73, 313-315. doi:10.1177/0190272510391422

Evans, M., Kelley, J. \& Kolosi, T. (1992). Images of class: Public perceptions in Hungary and Australia. American Sociological Review, 57, 461-482. doi:10.2307/2096095

Festinger, L. (1957). A theory of cognitive dissonance. Evanston, IL: Row, Peterson.

Fiske, S. T. \& Molm, L. D. (2010). Bridging inequality from both sides now. Social Psychology Quarterly, 73, 341-346. doi:10.1177/0190272510389007

Garretón, M. A. \& Cumsille, G. (2002). Las percepciones de la desigualdad en Chile. Proposiciones, 34, 1-9.

Gijsberts, M. (1999). The legitimation of inequality in state-socialist and market societies, 1987-1996. Amsterdam, Holanda: Thela Thesis.

Hojman, D. E. (1996). Poverty and inequality in Chile: Are democratic politics and neoliberal economics good for you? Journal of Interamerican Studies and World Affairs, 38(2/3), 73-96.

Howard, J. A. (1994). A social cognitive conception of social structure. Social Psychology Quarterly, 57, $210-227$. doi: $10.2307 / 2786877$

Huber, J. \& Form, W. H. (1973). Income and ideology: An analysis of the American political formula. New York, NY: Free Press.

Jost, J. T., Glaser, J., Kruglanski, A. W. \& Sulloway, F. J. (2003). Political conservatism as motivated social cognition. Psychological Bulletin, 129, 339-375. doi:10.1037/0033-2909.129.3.339

Jost, J. T., Pelham, B. W., Sheldon, O. \& Sullivan, B. N. (2003). Social inequality and the reduction of ideological dissonance on behalf of the system: Evidence of enhanced system justification among the disadvantaged. European Journal of Social Psychology, 33, 13-36. doi:10.1002/ejsp.127

Kelley, J. \& Evans, M. D. R. (1993). The legitimation of inequality: Occupational earnings in nine nations. American Journal of Sociology, 99, 75-125.

Kluegel, J. R. \& Smith, E. R. (1986). Beliefs about inequality: Americans'views of what is and what ought to be. New York, NY: Aldine de Gruyter.

Kluegel, J., Mason, D. \& Wegener, B. (1995). Social justice and political change: Public opinion in capitalist and postcommunist states. New York, NY: Aldine de Gruyter.

Lehmann, C. \& Hinzpeter, X. (2000). Los pobres no pueden esperar..., la desigualdad, sí. Los que más importan tienen la palabra (Puntos de Referencia $\mathrm{N}^{\circ}$ 233). Santiago, Chile: Centro de Estudios Públicos.

Lehmann, C. \& Hinzpeter, X. (2001). Los pobres no pueden esperar..., la desigualdad, sí. Los que más importan tienen la palabra (Parte II) (Puntos de Referencia No 241). Santiago, Chile: Centro de Estudios Públicos.

Levine, J. M., Resnick, L. B. \& Higgins, E. T. (1993). Social foundations of cognition. Annual Review of Psychology, 44, 585-612. doi:10.1146/annurev.ps.44.020193.003101

Melamed, D. \& North, M. S. (2010). The future in inequality. Social Psychology Quarterly, 73, 346-347. doi:10.1177/0 190272510389008

Mühleck, K. (2009). Gerechtigkeit und Wahlverhalten: Gerechtigkeitswahrnehmung und Gerechtigkeitseinstellungen als Motive politischen Handelns [Percepción de justicia y actitudes hacia la justicia como motivo de acción política]. Baden-Baden, Alemania: Nomos.

Ng, S. H. \& Allen, M. (2005). Perception of economic distributive justice: Exploring leading theories. Social Behavior and Personality, 33, 435-454.

Programa de Naciones Unidas para el Desarrollo (1998). Desarrollo humano en Chile: las paradojas de la modernización. Santiago, Chile: Autor.

Ruiz-Tagle, J. (1999). Chile: 40 años de desigualdad de ingresos. Santiago, Chile: Universidad de Chile, Departamento de Economía.

Runciman, W. G. (1966). Relative deprivation and social justice: A study of attitudes to social inequality in the twentieth century. London, Reino Unido: Routledge \& Kegan Paul.

Scalon, C. (2009, Julio). Percepção sobre desigualdades em perspectiva comparada. [Percepción de desigualdades desde una perspectiva comparada]. Ponencia presentada en el XXVIII International Congress of the Latin American Studies Association, Rio de Janeiro, Brasil.

Torche, F. (2005). Privatization reform and inequality of educational opportunity: The case of Chile. Sociology of Education, 78, 316-343. doi:10.1177/003804070507800403

United Nations Development Programme (2010). The real wealth of nations: Pathways to human development (Human Development Report 2010). New York, NY: Autor.

Wegener, B. (1987). The illusion of distributive justice. European Sociological Review, 3, 1-13.

Wegener, B. (1990). Equity, relative deprivation, and the value consensus paradox. Social Justice Research, 4, 65-86. doi:10.1007/BF01048536

Wegener, B. (1992). Concepts and measurement of prestige. Annual Review of Sociology, 18, 253-280. doi:10.1146/ annurev.so.18.080192.001345

Fecha de recepción: Mayo de 2011.

Fecha de aceptación: Marzo de 2012. 